\title{
ANALOGUE OF BOHL THEOREM FOR A CLASS OF LINEAR PARTIAL DIFFERENTIAL EQUATIONS
}

\author{
E. MUHAMADIEV, A.N. NAIMOV, A.Kh. SATTOROV
}

\begin{abstract}
We study the existence and uniqueness of a solution bounded in the entire space for a class of higher order linear partial differential equations. We prove the theorem on the necessary and sufficient condition for the existence and uniqueness of a bounded solution for a studied class of equations. This theorem is an analogue of the Bohl theorem known in the theory of ordinary differential equations. In a partial case the unique solvability conditions are expressed in terms of the coefficients of the equation and we provide the integral representation for the bounded solution.
\end{abstract}

Keywords: Bohl theorem, bounded solution, symbol of equation, representation of a bounded solution.

Mathematics Subject Classification: 35G05, 35E99, 35A01, 35A24, 35C15

\section{INTRODUCTION}

In the theory of ordinary differential equations, the Bohl theorem is known [1] on the unique solvability on the entire real axis $\mathbb{R}=(-\infty,+\infty)$ of the linear ordinary differential equation

$$
y^{(m)}+c_{1} y^{(m-1)}+\ldots+c_{m-1} y^{\prime}+c_{m} y=f(x), \quad x \in \mathbb{R},
$$

with constant coefficients $c_{1}, \ldots, c_{m}$ and the right hand side $f(x)$ continuous and bounded on $\mathbb{R}$. In accordance with Bohl theorem, for each continuous and bounded on $\mathbb{R}$ function $f(x)$, equation (1.1) has the unique bounded solution only in the case, when the symbol (characteristic polynomial) of the equation

$$
s^{m}+c_{1} s^{m-1}+\ldots+c_{m-1} s+c_{m},
$$

where $s=\sigma+i \tau$ is a complex variable, has no pure imaginary roots $i \tau, \tau \in \mathbb{R}$.

In the present work we formulate and prove an analogue of Bohl theorem for linear partial differential equations of the following form:

$$
\begin{aligned}
P_{1}\left(\frac{\partial}{\partial x_{1}}\right) \ldots P_{n}\left(\frac{\partial}{\partial x_{n}}\right) u & +\sum_{k_{1}=0}^{m_{1}-1} \ldots \sum_{k_{n}=0}^{m_{n}-1} b_{k_{1} \ldots k_{n}} \frac{\partial^{k_{1}+\ldots+k_{n}} u}{\partial x_{1}^{k_{1}} \ldots \partial x_{n}^{k_{n}}} \\
& =f\left(x_{1}, \ldots, x_{n}\right), \quad\left(x_{1}, \ldots, x_{n}\right) \in \mathbb{R}^{n} .
\end{aligned}
$$

Here we assume that we are given the natural numbers $n \geqslant 2, m_{1}, \ldots, m_{n}$, the complex numbers $b_{k_{1} \ldots k_{n}}, k_{j}=\overline{0, m_{j}-1}, j=\overline{1, n}$ and the polynomials

$$
P_{j}(s)=s^{m_{j}}+a_{j 1} s^{m_{j}-1}+\ldots+a_{j m_{j}}, \quad j=\overline{1, n},
$$

E.M. Muhamadiev, A.N. Naimov, A.Kh. Sattorov, Analogue of Bohl theorem for a Class of LINEAR PARTIAL DIFFERENTIAL EQUATIONS.

(c) Muhamadiev E.M., Naimov A.N., Sattorov A.Kh. 2017.

The work is partially supported by RFBR grants nos. 15-01-04713a, 16-01-00150a.

Submitted February 15, 2016. 
with constant complex coefficients $a_{j k}, k=\overline{1, m_{j}}, j=\overline{1, n}$. The function $f\left(x_{1}, \ldots, x_{n}\right)$ is assumed to be complex-valued, continuous and bounded in $\mathbb{R}^{n}$.

The issue on existence of bounded solutions to linear partial differential equations with constant coefficients was studied in work [2]. It was proved in this work that an arbitrary differential work of the form

$$
\sum_{k_{1}=0}^{m_{1}} \ldots \sum_{k_{n}=0}^{m_{n}} c_{k_{1} \ldots k_{n}} \frac{\partial^{k_{1}+\ldots+k_{n}} u}{\partial x_{1}^{k_{1}} \ldots \partial x_{n}^{k_{n}}}=f\left(x_{1}, \ldots, x_{n}\right), \quad\left(x_{1}, \ldots, x_{n}\right) \in \mathbb{R}^{n}
$$

with constant coefficients $c_{k_{1} \ldots k_{n}}$ is uniquely solvable in the space of bounded generalized functions if and only if the symbol of the equation

$$
\sum_{k_{1}=0}^{m_{1}} \ldots \sum_{k_{n}=0}^{m_{n}} c_{k_{1} \ldots k_{n}} s_{1}^{k_{1}} \ldots s_{n}^{k_{n}}
$$

where $s_{1}, \ldots, s_{n}$ are complex variables, has no pure imaginary roots $\left(i \tau_{1}, \ldots, i \tau_{n}\right), \tau_{j} \in \mathbb{R}$, $j=\overline{1, n}$. At that, if $f$ is a continuous and bounded in $\mathbb{R}^{n}$ function, then the solution $u$ is not necessarily continuous and bounded together with all its derivatives involved in the equation. As the theorems on hypoellipticity show [3], the smoothness of the solution depends on the behavior of the symbol of the equation at infinity. This is why it is an interesting issue on finding additional conditions for the symbol, apart from the absence of pure imaginary root, ensuring the smoothness of the solutions in the classical sense.

\section{MAIN RESUlTS}

For equations (1.2) we can formulate and prove the conditions for the unique solvability in the classical sense.

A bounded solution to equation $(1.2)$ is a complex-valued function $u\left(x_{1}, \ldots, x_{n}\right)$ continuous and bounded in $\mathbb{R}^{n}$ together with all partial derivatives

$$
\frac{\partial^{k_{1}+\ldots+k_{n}} u}{\partial x_{1}^{k_{1}} \ldots \partial x_{n}^{k_{n}}}, \quad \text { where } \quad k_{j}=\overline{0, m_{j}}, \quad j=\overline{1, n},
$$

which solves equation 1.2 .

In the present work we prove the following theorem.

Theorem 2.1. For each continuous and bounded in $\mathbb{R}^{n}$ function $f\left(x_{1}, \ldots, x_{n}\right)$, equation (1.2) has the unique bounded solution if and only if the polynomials $P_{1}, \ldots, P_{n}$ and the symbol of the equation

$$
P\left(s_{1}, \ldots, s_{n}\right) \equiv P_{1}\left(s_{1}\right) \cdot \ldots \cdot P_{n}\left(s_{n}\right)+P_{0}\left(s_{1}, \ldots, s_{n}\right),
$$

where

$$
P_{0}\left(s_{1}, \ldots, s_{n}\right)=\sum_{k_{1}=0}^{m_{1}-1} \ldots \sum_{k_{n}=0}^{m_{n}-1} b_{k_{1} \ldots k_{n}} s_{1}^{k_{1}} \ldots s_{n}^{k_{n}},
$$

have no pure imaginary roots, that is, for all $\tau_{1}, \ldots, \tau_{n} \in \mathbb{R}$, the conditions

$$
\begin{aligned}
& P\left(i \tau_{1}, \ldots, i \tau_{n}\right) \neq 0, \\
& P_{1}\left(i \tau_{1}\right) \neq 0, \quad \ldots, \quad P_{n}\left(i \tau_{n}\right) \neq 0,
\end{aligned}
$$

hold true. 
We note that in the case, when in equation $(1.2)$ all coefficients $b_{k_{1} \ldots k_{n}}$ are zero and conditions (2.2) are satisfied, the existence and the uniqueness of a bounded solution is implied by the Bohl theorem. One can confirm this by inverting one by one the differential operators

$$
P_{1}\left(\frac{\partial}{\partial x_{1}}\right), \quad \ldots, \quad P_{n}\left(\frac{\partial}{\partial x_{n}}\right) .
$$

Theorem 2.1 implies

Corollary 1. For each continuous and bounded in $\mathbb{R}^{2}$ function $f\left(x_{1}, x_{2}\right)$ the equation

$$
\begin{aligned}
\frac{\partial^{m_{1}+m_{2}} u}{\partial x_{1}^{m_{1}} \partial x_{2}^{m_{2}}}+ & \sum_{k_{1}=1}^{m_{1}} a_{1 k_{1}} \frac{\partial^{m_{1}-k_{1}+m_{2}} u}{\partial x_{1}^{m_{1}-k_{1}} \partial x_{2}^{m_{2}}}+\sum_{k_{2}=1}^{m_{2}} a_{2 k_{2}} \frac{\partial^{m_{1}+m_{2}-k_{2}} u}{\partial x_{1}^{m_{1}} \partial x_{2}^{m_{2}-k_{2}}} \\
& +\sum_{k_{1}=0}^{m_{1}-1} \sum_{k_{2}=0}^{m_{2}-1} b_{k_{1} k_{2}} \frac{\partial^{k_{1}+k_{2}} u}{\partial x_{1}^{k_{1}} \partial x_{2}^{k_{2}}}=f\left(x_{1}, x_{2}\right), \quad\left(x_{1}, x_{2}\right) \in \mathbb{R}^{2},
\end{aligned}
$$

with constant coefficients $a_{1 k_{1}}, a_{2 k_{2}}, b_{k_{1} k_{2}}$ has the unique bounded solution if and only if the symbol of the equation and the following two polynomials

$$
\begin{aligned}
& Q_{1}(s)=s^{m_{1}}+\sum_{k_{1}=1}^{m_{1}} a_{1 k_{1}} s^{m_{1}-k_{1}}, \\
& Q_{2}(s)=s^{m_{2}}+\sum_{k_{2}=1}^{m_{2}} a_{2 k_{2}} s^{m_{2}-k_{2}}
\end{aligned}
$$

have no pure imaginary roots.

We consider a particular case of equation (1.2), namely, the equation of the form

$$
\left(\frac{\partial}{\partial x_{1}}+a_{1}\right)^{m_{1}} \ldots\left(\frac{\partial}{\partial x_{n}}+a_{n}\right)^{m_{n}} u-b u=f\left(x_{1}, \ldots, x_{n}\right), \quad\left(x_{1}, \ldots, x_{n}\right) \in \mathbb{R}^{n},
$$

where the coefficients $a_{1}=\alpha_{1}+i \beta_{1}, \ldots, a_{n}=\alpha_{n}+i \beta_{n}$ and $b$ are complex numbers. The following theorems are true.

Theorem 2.2. For each continuous and bounded in $\mathbb{R}^{n}$ function $f\left(x_{1}, \ldots, x_{n}\right)$ equation (2.6) has the unique bounded solution if and only if the numbers $a_{1}=\alpha_{1}+i \beta_{1}, \ldots, a_{n}=\alpha_{n}+i \beta_{n}$ and $b$ satisfy the conditions

$$
\begin{aligned}
& \alpha_{1} \neq 0, \quad \ldots, \quad \alpha_{n} \neq 0, \\
& R_{\mid} m \mid\left(b \alpha_{1}^{-m_{1}} \ldots \alpha_{n}^{-m_{n}}\right)<1,
\end{aligned}
$$

where $|m|=m_{1}+\ldots+m_{n}$,

$$
R_{|m|}(c)=\max _{k=0, \ldots,|m|-1} \operatorname{Re}(\sqrt[|m|]{c})_{k}=|c|^{1 /|m|} \max _{k=0, \ldots,|m|-1} \cos \left(\frac{\arg (c)+2 \pi k}{|m|}\right) .
$$

Theorem 2.3. Assume that the numbers $\alpha_{1}, \ldots, \alpha_{n}$ are positive and condition (2.8) is satisfied. Then the unique bounded solution of equation (2.6) can be represented as

$$
u\left(x_{1}, \ldots, x_{n}\right)=\int_{-\infty}^{x_{1}} \ldots \int_{-\infty}^{x_{n}} G\left(x_{1}-\xi_{1}, \ldots, x_{n}-\xi_{n}\right) f\left(\xi_{1}, \ldots, \xi_{n}\right) d \xi_{1} \ldots d \xi_{n},
$$

where the function $G\left(x_{1}, \ldots, x_{n}\right)$ is defined by the formula

$$
G\left(x_{1}, \ldots, x_{n}\right)=e^{-a_{1} x_{1}-\ldots-a_{n} x_{n}} \sum_{k=0}^{\infty} \frac{x_{1}^{m_{1}-1} \ldots x_{n}^{m_{n}-1}\left(b x_{1}^{m_{1}} \ldots x_{n}^{m_{n}}\right)^{k}}{\left(m_{1}(k+1)-1\right) ! \ldots\left(m_{n}(k+1)-1\right) !}
$$


and is absolutely integrable in the domain $x_{1}>0, \ldots, x_{n}>0$ :

$$
\int_{0}^{+\infty} \ldots \int_{0}^{+\infty}\left|G\left(x_{1}, \ldots, x_{n}\right)\right| d x_{1} \ldots d x_{n}<\infty .
$$

Remark 1. Under the change of $x_{j}$ by $y_{j}=-x_{j}$, the bracket $\left(\partial / \partial x_{j}+a_{j}\right)^{m_{j}}$ is transformed into the bracket $(-1)^{m_{j}}\left(\partial / \partial y_{j}-a_{j}\right)^{m_{j}}$. Therefore, equation (2.6) with nonzero $\alpha_{1}, \ldots, \alpha_{n}$ can be always reduced to the case when $\alpha_{1}, \ldots, \alpha_{n}$ are positive.

Theorems 2.2 and 2.3 for $n=2$ and $m_{1}=m_{2}=1$ were proved in works [4], [5]. The monograph [6] provides results on exponential representation of generalized solutions to linear differential equations with constant coefficients. The results of this monograph do not imply Theorems 2.1 2.3 .

In our opinion, the obtained results can be extended for partial differential equations with variable coefficients by proving and applying Carleman type inequalities ([7]).

\section{EXISTENCE AND UNIQUENESS OF BOUNDED SOLUTION}

In this section we prove Theorem 2.1. First we introduce the notations: $m=\left(m_{1}, \ldots, m_{n}\right)$ is the vector formed by the degrees $m_{1}, \ldots, m_{n}$ of the polynomials $P_{1}, \ldots, P_{n},|m|=m_{1}+\ldots+m_{n}$, $C_{0}$ is the Banach space of continuous and bounded in $\mathbb{R}^{n}$ functions $v\left(x_{1}, \ldots, x_{n}\right)$ with the norm

$$
\|v\|=\sup _{\left(x_{1}, \ldots, x_{n}\right) \in \mathbb{R}^{n}}\left|v\left(x_{1}, \ldots, x_{n}\right)\right|
$$

$C_{m}$ is the Banach space of functions $v\left(x_{1}, \ldots, x_{n}\right)$, belonging to $C_{0}$ together with all its derivatives

$$
\frac{\partial^{k_{1}+\ldots+k_{n}} v}{\partial x_{1}^{k_{1}} \ldots \partial x_{n}^{k_{n}}}, \quad k_{j}=\overline{0, m_{j}}, \quad j=\overline{1, n},
$$

where the norm is defined by the formula

$$
\|v\|_{m}=\sum_{k_{j}=\overline{0, m_{j}}, j=\overline{1, n}}\left\|\frac{\partial^{k_{1}+\ldots+k_{n}} v}{\partial x_{1}^{k_{1}} \ldots \partial x_{n}^{k_{n}}}\right\|,
$$

$S$ is the space of functions $v\left(x_{1}, \ldots, x_{n}\right)$ infinitely differentiable in $\mathbb{R}^{n}$ and fast decaying at infinity [8], $S^{\prime}$ is the space of tempered distributions [8]. The embeddings $S \subset C_{m} \subset C_{0} \subset S^{\prime}$ hold true [8].

Before proving Theorem 2.1, let us check three lemmata.

Lemma 3.1. If condition (2.1) is satisfied, then a solution of equation (1.2) is unique in the space $S^{\prime}$.

Proof. Let $f=0$ and $u \in S^{\prime}$ in equation 1.2 . We apply the Fourier transform of the generalized functions [8] to the both sides of $(1.2)$ and obtain the identity

$$
\left(\widehat{u}, P^{\prime} \psi\right)=0 \quad \text { for each } \quad \psi \in S \text {. }
$$

Here $\widehat{u}$ is the Fourier image of $u, \widehat{u} \in S^{\prime}, P^{\prime}=P\left(-i \tau_{1}, \ldots,-i \tau_{n}\right)$. It follows from condition (2.1) that $\varphi / P^{\prime} \in S$ for each compactly supported function $\varphi \in S$. Letting $\psi=\varphi / P^{\prime}$ in the identity (3.1), we obtain $(\widehat{u}, \varphi)=0$ for each compactly supported function $\varphi \in S$. The set of compactly supported functions is dense in $S$ [8] and therefore, $\widehat{u}=0$ and $u=0$. The proof is complete.

Lemma 3.2. Assume that conditions (2.1), (2.2) are satisfied and for some $f \in C_{0}$ there exists a solution $u$ to equation (1.2) in $S^{\prime}$ such that $P_{0}\left(\partial / \partial x_{1}, \ldots, \partial / \partial x_{n}\right) u \in C_{0}$. Then $u \in C_{m}$. 
Proof. Given $f$ and $u$, we consider the equation

$$
P_{1}\left(\frac{\partial}{\partial x_{1}}\right) \ldots P_{n}\left(\frac{\partial}{\partial x_{n}}\right) v=g
$$

where

$$
g=f-P_{0}\left(\frac{\partial}{\partial x_{1}}, \ldots, \frac{\partial}{\partial x_{n}}\right) u \in C_{0} .
$$

On one hand, $u$ is a generalized solution to equation (3.2). On the other hand, we can apply $n$ times Bohl theorem [1] to equation (3.2) and invert the differential operators

$$
P_{1}\left(\frac{\partial}{\partial x_{1}}\right), \quad \ldots, \quad P_{n}\left(\frac{\partial}{\partial x_{n}}\right) .
$$

While inverting each differential operator $P_{j}\left(\partial / \partial x_{j}\right)$, we keep the property of being bounded and continuous for partial derivatives w.r.t. other variables. As a result we obtain a solution $v \in C_{m}$ of equation (3.2). Equation $(3.2)$ is a particular case of equation $(1.2)$ and by Lemma 3.1 it can have only the unique solution in $S^{\prime}$. Therefore, $u=v$. The proof is complete.

Lemma 3.3. If conditions (2.1) and (2.2) are satisfied, there exists a positive number $\gamma$ such that for all $\tau_{1}, \ldots, \tau_{n} \in \mathbb{R}$ the estimate

$$
\left|P\left(i \tau_{1}, \ldots, i \tau_{n}\right)\right| \geqslant \gamma\left(1+\left|\tau_{1}\right|\right)^{m_{1}} \ldots\left(1+\left|\tau_{n}\right|\right)^{m_{n}}
$$

holds true.

Proof. For each $j=\overline{1, n}$ we expand the polynomial $P_{j}(s)$ over its roots

$$
P_{j}(s)=\left(s-\lambda_{j 1}\right) \ldots\left(s-\lambda_{j m_{j}}\right) .
$$

We have

$$
\frac{\left|P_{j}\left(i \tau_{j}\right)\right|}{\left(1+\left|\tau_{j}\right|\right)^{m_{j}}}=\prod_{k=1}^{m_{j}}\left|\frac{i \tau_{j}-\lambda_{j k}}{1+\left|\tau_{j}\right|}\right| \rightarrow 1 \quad \text { as } \quad \tau_{j} \rightarrow \infty,
$$

and by conditions 2.2 we obtain:

$$
\inf _{\tau_{j} \in \mathbb{R}} \frac{\left|P_{j}\left(i \tau_{j}\right)\right|}{\left(1+\left|\tau_{j}\right|\right)^{m_{j}}}=\gamma_{j}>0 .
$$

Therefore, for all $\tau_{1}, \ldots, \tau_{n} \in \mathbb{R}$ we have the estimate

$$
\frac{\left|P_{1}\left(i \tau_{1}\right) \ldots P_{n}\left(i \tau_{n}\right)\right|}{\left(1+\left|\tau_{1}\right|\right)^{m_{1}} \ldots\left(1+\left|\tau_{n}\right|\right)^{m_{n}}} \geqslant \gamma_{1} \ldots \gamma_{n}=2 \widetilde{\gamma}_{1} .
$$

For the polynomial $P_{0}$ we get

$$
\left|P_{0}\left(i \tau_{1}, \ldots, i \tau_{n}\right)\right| \leqslant c_{0}\left(1+\left|\tau_{1}\right|\right)^{m_{1}-1} \ldots\left(1+\left|\tau_{n}\right|\right)^{m_{n}-1},
$$

where $c_{0}$ is independent of $\tau_{1}, \ldots, \tau_{n}$. This yields

$$
\frac{\left|P\left(i \tau_{1}, \ldots, i \tau_{n}\right)\right|}{\left(1+\left|\tau_{1}\right|\right)^{m_{1}} \ldots\left(1+\left|\tau_{n}\right|\right)^{m_{n}}} \geqslant 2 \widetilde{\gamma}_{1}-\frac{c_{0}}{\left(1+\left|\tau_{1}\right|\right) \ldots\left(1+\left|\tau_{n}\right|\right)}>\widetilde{\gamma}_{1}
$$

as $\left|\tau_{1}\right|+\ldots+\left|\tau_{n}\right|>c_{0} / \widetilde{\gamma}_{1}$. By condition (2.1) we obtain:

$$
\min _{\left|\tau_{1}\right|+\ldots+\left|\tau_{n}\right| \leqslant c_{0} / \widetilde{\gamma}_{1}} \frac{\left|P\left(i \tau_{1}, \ldots, i \tau_{n}\right)\right|}{\left(1+\left|\tau_{1}\right|\right)^{m_{1}} \ldots\left(1+\left|\tau_{n}\right|\right)^{m_{n}}}=\widetilde{\gamma}_{2}>0 .
$$

Hence, for all $\tau_{1}, \ldots, \tau_{n} \in \mathbb{R}$ estimate $(3.3)$ holds true, where $\gamma$ is the smallest of the numbers $\widetilde{\gamma}_{1}$ and $\widetilde{\gamma}_{2}$. The proof is complete.

We proceed to the proof of Theorem 2.1. 
Proof of Theorem 2.1. Necessity. Assume that for each $f \in C_{0}$ equation (1.2) has the unique solution $u \in C_{m}$. Then condition (2.1) should be satisfied. Indeed, if $P\left(i \tau_{1}^{0}, \ldots, i \tau_{n}^{0}\right)=0$ for some $\tau_{1}^{0}, \ldots, \tau_{n}^{0} \in \mathbb{R}$, then the function $u+\exp \left(i \tau_{1}^{0} x_{1}+\ldots+i \tau_{n}^{0} x_{n}\right)$ also is a solution of equation (1.2) in $C_{m}$.

Assume that one of conditions 2.2 fails, for instance, $P_{1}\left(i \tau_{1}^{0}\right)=0$ for some $\tau_{1}^{0} \in \mathbb{R}$. We take some function $v^{0}\left(x_{2}, \ldots, x_{n}\right) \in C_{m^{\prime \prime}} \backslash C_{m^{\prime}}$, where $m^{\prime}=\left(m_{2}, m_{3}, \ldots, m_{n}\right), m^{\prime \prime}=$ $\left(m_{2}-1, m_{3}, \ldots, m_{n}\right)$, and consider the function $u^{0}\left(x_{1}, \ldots, x_{n}\right)=\exp \left(i \tau_{1}^{0} x_{1}\right) v^{0}\left(x_{2}, \ldots, x_{n}\right)$. It is obvious that $u^{0} \in S^{\prime} \backslash C_{m}$ and

$$
P\left(\frac{\partial}{\partial x_{1}}, \ldots, \frac{\partial}{\partial x_{n}}\right) u^{0}=P_{0}\left(\frac{\partial}{\partial x_{1}}, \ldots, \frac{\partial}{\partial x_{n}}\right) u^{0} \equiv f^{0} \in C_{0} .
$$

Therefore, the function $u^{0}$ is a generalized solution to equation 1.2 in $S^{\prime}$ as $f=f^{0}$. Since condition (2.1) is satisfied, by Lemma 3.1, equation (1.2) can have only the unique solution in the space $S^{\prime}$. Thus, as $f=f^{0}$, equation (1.2) has no solutions in $C_{m}$, which is a contradiction. Hence, conditions (2.2) are necessary.

Sufficiency. Assume that conditions (2.1) and (2.2) hold true. We first prove the solvability of equation 1.2 for periodic functions $f$ in $C_{0}$. We shall call function $f$ periodic with a period $\omega$, where $\omega$ is a fixed positive number, if for each $j=\overline{1, n}$ the identity $f\left(x_{1}, \ldots, x_{j}+\omega, \ldots, x_{n}\right) \equiv$ $f\left(x_{1}, \ldots, x_{j}, \ldots, x_{n}\right)$ holds true.

Lemma 3.4. For each $\omega$-periodic function $f$ in $C_{0}$ there exists the unique solution $u$ of equation (1.2) in $C_{m}$, which $\omega$-periodic together with all its derivatives of order up to $m$. At that, the estimate

$$
\|u\|_{m} \leqslant M_{0}\|f\|
$$

holds true, where $M_{0}$ is a positive number independent of $f$ and $\omega$ for all $\omega \geqslant 1$.

Proof. We shall seek a periodic solution as the Fourier series of the exponentials [9]. In order to do it, we expand $\omega$-periodic function $f$ into the Fourier series

$$
f=\sum_{\left(l_{1}, \ldots, l_{n}\right)} c_{l_{1} \ldots l_{n}}(f) e^{i \frac{2 \pi}{\omega}\left(l_{1} x_{1}+\ldots+l_{n} x_{n}\right)} .
$$

The series converges in the norm of the space $L_{2}\left(D_{\omega}\right)([9])$

$$
\|g\|_{L_{2}\left(D_{\omega}\right)}^{2}=\frac{1}{\omega^{n}} \int_{0}^{\omega} \ldots \int_{0}^{\omega}\left|g\left(\xi_{1}, \ldots, \xi_{n}\right)\right|^{2} d \xi_{1} \ldots d \xi_{n}
$$

where $D_{\omega}=\left\{\left(\xi_{1}, \ldots, \xi_{n}\right): 0<\xi_{j}<\omega, j=\overline{1, n}\right\}$, and the Parseval identity

$$
\sum_{\left(l_{1}, \ldots, l_{n}\right)}\left|c_{l_{1} \ldots l_{n}}(f)\right|^{2}=\|f\|_{L_{2}\left(D_{\omega}\right)}^{2}
$$

holds true. A periodic solution to equation 1.2 can be defined by the formulae

$$
\begin{aligned}
& u=\sum_{\left(l_{1}, \ldots, l_{n}\right)} c_{l_{1} \ldots l_{n}}(u) e^{i \frac{2 \pi}{\omega}\left(l_{1} x_{1}+\ldots+l_{n} x_{n}\right)}, \\
& c_{l_{1} \ldots l_{n}}(u)=\frac{c_{l_{1} \ldots l_{n}}(f)}{P\left(i \frac{2 \pi}{\omega} l_{1}, \ldots, i \frac{2 \pi}{\omega} l_{n}\right)} .
\end{aligned}
$$

Taking into consideration Lemma 3.3 , we get the following estimates as $0 \leqslant k_{j}<m_{j}, j=\overline{1, n}$ :

$$
\left|\left(i \frac{2 \pi}{\omega} l_{1}\right)^{k_{1}} \ldots\left(i \frac{2 \pi}{\omega} l_{n}\right)^{k_{n}} c_{l_{1} \ldots l_{n}}(u)\right| \leqslant \frac{\left|\frac{2 \pi}{\omega} l_{1}\right|^{k_{1}} \ldots\left|\frac{2 \pi}{\omega} l_{n}\right|^{k_{n}}\left|c_{l_{1} \ldots l_{n}}(f)\right|}{\gamma\left(1+\left|\frac{2 \pi}{\omega} l_{1}\right|\right)^{m_{1}} \ldots\left(1+\left|\frac{2 \pi}{\omega} l_{n}\right|\right)^{m_{n}}}
$$




$$
\leqslant \frac{1}{\gamma}\left(1+\left|\frac{2 \pi}{\omega} l_{1}\right|\right)^{-1} \ldots\left(1+\left|\frac{2 \pi}{\omega} l_{n}\right|\right)^{-1}\left|c_{l_{1} \ldots l_{n}}(f)\right|
$$

and by Cauchy-Schwarz inequality [10]

$$
\begin{aligned}
\sum_{\left(l_{1}, \ldots, l_{n}\right)} \mid & \left(i \frac{2 \pi}{\omega} l_{1}\right)^{k_{1}} \ldots\left(i \frac{2 \pi}{\omega} l_{n}\right)^{k_{n}} c_{l_{1} \ldots l_{n}}(u) \mid \\
& \leqslant \frac{1}{\gamma}\left(\sum_{\left(l_{1}, \ldots, l_{n}\right)}\left(1+\left|\frac{2 \pi}{\omega} l_{1}\right|\right)^{-2} \ldots\left(1+\left|\frac{2 \pi}{\omega} l_{n}\right|\right)^{-2}\right)^{1 / 2}\left(\sum_{\left(l_{1}, \ldots, l_{n}\right)}\left|c_{l_{1} \ldots l_{n}}(f)\right|^{2}\right)^{1 / 2} .
\end{aligned}
$$

This implies, first, that $u \in C_{m^{\prime}}$, where $m^{\prime}=\left(m_{1}-1, \ldots, m_{n}-1\right)$, and second, that the function $u$ and all its partial derivatives $\partial^{k_{1}+\ldots+k_{n}} u / \partial x_{1}^{k_{1}} \ldots \partial x_{n}^{k_{n}}$, where $k_{j}=\overline{0, m_{j}-1}, j=\overline{1, n}$, are $\omega$-periodic, and third, the estimate

$$
\|u\|_{m^{\prime}} \leqslant M_{1}\|f\|_{L_{2}\left(D_{\omega}\right)} \leqslant M_{1}\|f\|
$$

holds true. Here $M_{1}>0$ and $M_{1}$ is independent of $f$ and $\omega$ for all $\omega \geqslant 1$. It follows from estimate (3.7) and formulae (3.5), (3.6) that $u \in S^{\prime}$ and $u$ is a generalized solution of equation (1.2) and $P_{0}\left(\partial / \partial x_{1}, \ldots, \partial / \partial x_{n}\right) u \in C_{0}$. Applying Lemma 3.2, we get that $u \in C_{m}$ and the estimate

$$
\|u\|_{m} \leqslant M_{2}\|\tilde{f}\|
$$

is valid, where $\tilde{f}=f-P_{0}\left(\partial / \partial x_{1}, \ldots, \partial / \partial x_{n}\right) u$. By (3.7) and (3.8) we obtain estimate (3.4). The proof is complete.

Let $f$ be an arbitrary function in $C_{0}$. We construct the following sequence of periodic functions:

$$
\begin{aligned}
& f_{q}\left(x_{1}, \ldots, x_{n}\right)=f\left(x_{1}, \ldots, x_{n}\right) \quad \text { as } \quad \max \left(\left|x_{1}\right|, \ldots,\left|x_{n}\right|\right) \leqslant q, \\
& f_{q}\left(x_{1}, \ldots, x_{n}\right)=\eta_{q}\left(\max \left(\left|x_{1}\right|, \ldots,\left|x_{n}\right|\right)\right) f\left(x_{1}, \ldots, x_{n}\right) \quad \text { as } \quad q<\max \left(\left|x_{1}\right|, \ldots,\left|x_{n}\right|\right) \leqslant q+1,
\end{aligned}
$$

where $q=1,2, \ldots,\left(x_{1}, \ldots, x_{n}\right) \in D_{q}, D_{q}=\left\{\left(\xi_{1}, \ldots, \xi_{n}\right):\left|\xi_{j}\right| \leqslant q+1, j=\overline{1, n}\right\}$, the function $\eta_{q}(t)$ is continuous on $\mathbb{R}, 0 \leqslant \eta_{q}(t) \leqslant 1$ for all $t, \eta_{q}(t)=1$ as $|t| \leqslant q$ and $\eta_{q}(t)=0$ as $|t|>q+1$. We continue each function $f_{q}$ outside $D_{q}$ periodically with the period $2(q+1)$. It is obvious that $\left\|f_{q}\right\| \leqslant\|f\|$ for all $q=1,2, \ldots$ and $f_{q} \rightarrow f$ as $q \rightarrow \infty$ uniformly in each bounded set $D \in \mathbb{R}^{n}$. In accordance with Lemma 3.4 for each $q$ there exists the unique solution $u_{q}$ in $C_{m}$ of equation (1.2) and the estimate $\left\|u_{q}\right\|_{m} \leqslant M_{0}\|f\|$ holds true. This estimate, by Arzelà-Ascoli theorem [10], there exists a subsequence $u_{q_{1}}, u_{q_{2}}, \ldots$ converging uniformly in each bounded set $D \in \mathbb{R}^{n}$ to some function $\widetilde{u} \in C_{m^{\prime}}\left(m^{\prime}=\left(m_{1}-1, \ldots, m_{n}-1\right)\right)$ together with all partial derivatives $\partial^{k_{1}+\ldots+k_{n}} / \partial x_{1}^{k_{1}} \ldots \partial x_{n}^{k_{n}}$, where $k_{j}=\overline{0, m_{j}-1}, j=\overline{1, n}$.

In equation (1.2) we let $f=f_{q_{j}}, u=u_{q_{j}}$ and pass to the limit as $j \rightarrow \infty$ in the space of generalized functions. Then we obtain that $\widetilde{u}$ is a generalized solution to equation (1.2) and satisfy assumptions of Lemma 3.2. According to Lemma 3.2, we have $\widetilde{u} \in C_{m}$. The proof of Theorem 2.1 is complete.

Proof Corollary 1. Let us show that Theorem 2.1 implies Corollary 1. In order to prove this, it is sufficient to confirm that equation (2.3) can be represented as (1.2). As $P_{1}\left(s_{1}\right)$ and $P_{2}\left(s_{2}\right)$, we consider the following polynomials

$$
\begin{aligned}
& P_{1}\left(s_{1}\right)=\left(s_{1}+z_{11}\right) \cdot \ldots \cdot\left(s_{1}+z_{1 m_{1}}\right) \equiv(-1)^{m_{1}} Q_{1}\left(s_{1}\right), \\
& P_{2}\left(s_{2}\right)=\left(s_{2}+z_{21}\right) \cdot \ldots \cdot\left(s_{2}+z_{2 m_{2}}\right) \equiv(-1)^{m_{2}} Q_{2}\left(s_{2}\right),
\end{aligned}
$$


where $-z_{11}, \ldots,-z_{1 m_{1}}$ are the roots of the polynomial $Q_{1}(s),-z_{21}, \ldots,-z_{2 m_{2}}$ are the roots of the polynomial $Q_{2}(s)$. Multiplying the polynomials $P_{1}\left(s_{1}\right)$ and $P_{2}\left(s_{2}\right)$ and employing Vieta's formulae expressing the coefficients of a polynomial in terms of its roots [11], we get

$$
\begin{aligned}
P_{1}\left(s_{1}\right) & P_{2}\left(s_{2}\right)=s_{1}^{m_{1}} s_{2}^{m_{2}}+\sum_{k_{1}=0}^{m_{1}-1} \sum_{k_{2}=0}^{m_{2}-1} \widetilde{b}_{k_{1} k_{2}} s_{1}^{k_{1}} s_{2}^{k_{2}} \\
& +s_{2}^{m_{2}}\left(\left(z_{11}+\ldots+z_{1 m_{1}}\right) s_{1}^{m_{1}-1}+\left(z_{11} z_{12}+\ldots+z_{1 m_{1}-1} z_{1 m_{1}}\right) s_{1}^{m_{1}-2} \ldots+z_{11} \ldots z_{1 m_{1}}\right) \\
& +s_{1}^{m_{1}}\left(\left(z_{21}+\ldots+z_{2 m_{2}}\right) s_{2}^{m_{2}-1}+\left(z_{21} z_{22}+\ldots+z_{2 m_{2}-1} z_{2 m_{2}}\right) s_{2}^{m_{2}-2}+\ldots+z_{21} \ldots z_{2 m_{2}}\right) \\
= & s_{1}^{m_{1}} s_{2}^{m_{2}}+s_{2}^{m_{2}} \sum_{k_{1}=1}^{m_{1}} a_{1 k_{1}} s_{1}^{m_{1}-k_{1}}+s_{1}^{m_{1}} \sum_{k_{2}=1}^{m_{2}} a_{2 k_{2}} s_{2}^{m_{2}-k_{2}}+\sum_{k_{1}=0}^{m_{1}-1} \sum_{k_{2}=0}^{m_{2}-1} \widetilde{b}_{k_{1} k_{2}} s_{1}^{k_{1}} s_{2}^{k_{2}} .
\end{aligned}
$$

Therefore, the symbol $P\left(s_{1}, s_{2}\right)$ of equation 2.3$)$ can be represented as

$$
P\left(s_{1}, s_{2}\right)=P_{1}\left(s_{1}\right) P_{2}\left(s_{2}\right)+\sum_{k_{1}=0}^{m_{1}-1} \sum_{k_{2}=0}^{m_{2}-1}\left(b_{k_{1} k_{2}}-\widetilde{b}_{k_{1} k_{2}}\right) s_{1}^{k_{1}} s_{2}^{k_{2}} .
$$

The polynomials $P_{1}$ and $P_{2}$ have no pure imaginary roots simultaneously with the polynomials $Q_{1}$ and $Q_{2}$. Hence, equation $(2.3)$ can be represented as (1.2) and the assumptions of Theorem 2.1 are satisfied only in the case, when the symbol of equation (2.3) and the polynomials $Q_{1}, Q_{2}$ have no pure imaginary roots. The proof is complete.

\section{Proof of Theorem 2.2}

Theorem 2.2 is implied by Theorem 2.1 and the following lemma.

Lemma 4.1. If conditions (2.7) are satisfied, then the symbol of equation (2.6) has no pure imaginary roots only in the case, when condition (2.8) holds true.

Proof. It is easy to check that the condition on the absence of pure imaginary roots for the symbol of equation 2.6 is equivalent to the condition

$$
\left(i \tau_{1}+1\right)^{m_{1}} \ldots\left(i \tau_{n}+1\right)^{m_{n}} \neq \widetilde{b} \quad \text { for all } \tau_{1}, \ldots, \tau_{n} \in \mathbb{R},
$$

where $\widetilde{b}=b \alpha_{1}^{-m_{1}} \ldots \alpha_{n}^{-m_{n}}$. Let us show that condition 4.1 is equivalent to the condition

$$
R_{|m|}(\widetilde{b})<1,
$$

where $|m|=m_{1}+\ldots+m_{n}$, and $R_{|m|}(\widetilde{b})$ is determined by the formula $(2.9)$. This will prove Lemma 4.1.

We let $\tau_{j}=\operatorname{tg} \varphi_{j}$, where $\varphi_{j} \in(-\pi / 2, \pi / 2)$ for all $j=\overline{1, n}$. Then condition 4.1 becomes

$$
e^{i\left(m_{1} \varphi_{1}+\ldots+m_{n} \varphi_{n}\right)} \neq \widetilde{b} \cos ^{m_{1}} \varphi_{1} \ldots \cos ^{m_{n}} \varphi_{n} \quad \text { for all } \varphi_{1}, \ldots, \varphi_{n} \in\left(-\frac{\pi}{2}, \frac{\pi}{2}\right) \text {. }
$$

In its turn, this is equivalent to the condition:

$$
|\widetilde{b}| \cos ^{m_{1}} \varphi_{1} \ldots \cos ^{m_{n}} \varphi_{n} \neq 1
$$

for all $\varphi_{1}, \ldots, \varphi_{n} \in(-\pi / 2, \pi / 2)$ satisfying the condition $m_{1} \varphi_{1}+\ldots+m_{n} \varphi_{n}=\theta+2 \pi l$ for some integer $l$ and $\theta=\arg \widetilde{b}$, which is the argument of $\widetilde{b},|\theta| \leqslant \pi$. It is obvious that the integer number $l$ should satisfy the inequality $|\theta+2 \pi l|<|m| \pi / 2$.

Let $\left(\varphi_{1}^{0}, \ldots, \varphi_{n}^{0}\right)$ be a set satisfying the aforementioned conditions for some integer $l_{0}$, on which the extremum of the function is attained $F\left(\varphi_{1}, \ldots, \varphi_{n}\right)=\cos ^{m_{1}} \varphi_{1} \ldots \cos ^{m_{n}}$. Let us check that the inequalities

$$
|\widetilde{b}| \cos ^{m_{1}} \varphi_{1}^{0} \ldots \cos ^{m_{n}} \varphi_{n}^{0}<1
$$


and the identities

$$
\varphi_{1}^{0}=\ldots=\varphi_{n}^{0}=\frac{\theta+2 \pi l_{0}}{|m|}
$$

hold true. If so, then condition 4.3 would be equivalent the inequality

$$
|\widetilde{b}|^{1 /|m|} \cos \frac{\theta+2 \pi l_{0}}{|m|}<1,
$$

which in notations $(2.9)$ become $(4.2)$ and this would complete the proof of the lemma.

Before we check inequality 4.4$)$, we construct the set $\left(\widetilde{\varphi}_{1}, \ldots, \widetilde{\varphi}_{n}\right)$ satisfying the conditions

$$
\left(\widetilde{\varphi}_{1}, \ldots, \widetilde{\varphi}_{n}\right) \in\left(-\frac{\pi}{2}, \frac{\pi}{2}\right), \quad m_{1} \widetilde{\varphi}_{1}+\ldots+m_{n} \widetilde{\varphi}_{n}=\theta+2 \pi l_{0}, \quad|\widetilde{b}| F\left(\widetilde{\varphi}_{1}, \ldots, \widetilde{\varphi}_{n}\right)<1 .
$$

At that, without loss of generality, we can assume that $\theta+2 \pi l_{0} \geqslant 0 \quad m_{1} \leqslant|m| / 2$. We choose $\delta \in(0, \pi / 2)$ so that the inequalities

$$
|\widetilde{b}| \cos ^{m_{1}}\left(\frac{\pi}{2}-\delta\right)<1, \quad 0<\theta+2 \pi l_{0}+m_{1} \delta<\frac{|m| \pi}{2}
$$

are satisfied. We let $\widetilde{\varphi}_{1}=\pi / 2-\delta$. For $\widetilde{\varphi}_{1}$ we have the belonging

$$
\theta+2 \pi l_{0}-m_{1} \widetilde{\varphi}_{1} \in\left(-\left(|m|-m_{1}\right) \frac{\pi}{2} \quad, \quad\left(|m|-m_{1}\right) \frac{\pi}{2}\right) .
$$

Indeed, by the assumptions $\theta+2 \pi l_{0} \geqslant 0, m_{1} \leqslant|m| / 2$ and the choice of $\delta$, we have

$$
\begin{aligned}
& \theta+2 \pi l_{0}-m_{1} \widetilde{\varphi}_{1} \geqslant-m_{1} \frac{\pi}{2}+m_{1} \delta>-\left(|m|-m_{1}\right) \frac{\pi}{2}, \\
& \theta+2 \pi l_{0}-m_{1} \widetilde{\varphi}_{1}=\theta+2 \pi l_{0}+m_{1} \delta-m_{1} \frac{\pi}{2}<\left(|m|-m_{1}\right) \frac{\pi}{2} .
\end{aligned}
$$

By 4.6) we obtain the existence of $\widetilde{\varphi}_{2}, \ldots, \widetilde{\varphi}_{n} \in(-\pi / 2, \pi / 2)$ such that

$$
m_{2} \widetilde{\varphi}_{2}+\ldots+m_{n} \widetilde{\varphi}_{n}=\theta+2 \pi l_{0}-m_{1} \widetilde{\varphi}_{1} .
$$

For the set $\left(\widetilde{\varphi}_{1}, \ldots, \widetilde{\varphi}_{n}\right)$ we have

$$
|\widetilde{b}| F\left(\widetilde{\varphi}_{1}, \ldots, \widetilde{\varphi}_{n}\right) \leqslant|\widetilde{b}| \cos ^{m_{1}}\left(\frac{\pi}{2}-\delta\right)<1 .
$$

By two sets $\left(\varphi_{1}^{0}, \ldots, \varphi_{n}^{0}\right)$ and $\left(\widetilde{\varphi}_{1}, \ldots, \widetilde{\varphi}_{n}\right)$ we introduce the function

$$
g(t)=|\widetilde{b}| F\left((1-t) \widetilde{\varphi}_{1}+t \varphi_{1}^{0}, \ldots,(1-t) \widetilde{\varphi}_{n}+t \varphi_{n}^{0}\right), \quad t \in[0,1] .
$$

The function $g(t)$ is continuous on the segment $[0,1]$ and $g(0)<1$. If 4.4$)$ fails, then $g(1)>1$ and therefore, for some $t_{0} \in(0,1)$ we should have $g\left(t_{0}\right)=1$. For this value $t_{0}$ we get

$$
\begin{aligned}
& \left(1-t_{0}\right) \widetilde{\varphi}_{1}+t_{0} \varphi_{1}^{0}, \ldots,\left(1-t_{0}\right) \widetilde{\varphi}_{n}+t_{0} \varphi_{n}^{0} \in\left(-\frac{\pi}{2}, \frac{\pi}{2}\right), \\
& m_{1}\left[\left(1-t_{0}\right) \widetilde{\varphi}_{1}+t_{0} \varphi_{1}^{0}\right]+\ldots+m_{n}\left[\left(1-t_{0}\right) \widetilde{\varphi}_{n}+t_{0} \varphi_{n}^{0}\right]=\theta+2 \pi l_{0}, \\
& |\widetilde{b}| F\left(\left(1-t_{0}\right) \widetilde{\varphi}_{1}+t_{0} \varphi_{1}^{0}, \ldots,\left(1-t_{0}\right) \widetilde{\varphi}_{n}+t_{0} \varphi_{n}^{0}\right)=1
\end{aligned}
$$

This contradicts condition (4.3). Therefore, (4.4) indeed holds true.

The set $\left(\varphi_{1}^{0}, \ldots, \varphi_{n-1}^{0}\right)$ is the point of the maximum of the function

$$
F_{1}\left(\varphi_{1}, \ldots, \varphi_{n-1}\right) \equiv F\left(\varphi_{1}, \ldots, \varphi_{n-1}, \frac{1}{m_{n}}\left(\theta+2 \pi l_{0}-m_{1} \varphi_{1}-\ldots m_{n-1} \varphi_{n-1}\right)\right)
$$

and it lies inside the domain, in which the function $F_{1}$ is considered. This is why by the extremum necessary condition we obtain:

$$
\frac{\partial F_{1}}{\partial \varphi_{j}}\left(\varphi_{1}^{0}, \ldots, \varphi_{n-1}^{0}\right)=0, \quad j=\overline{1, n-1}
$$




$$
\sin \left(\frac{1}{m_{n}}\left(\theta+2 \pi l_{0}-m_{1} \varphi_{1}^{0}-\ldots m_{n-1} \varphi_{n-1}^{0}\right)-\varphi_{j}^{0}\right)=0, \quad j=\overline{1, n-1}
$$

where

$$
\begin{aligned}
& \frac{1}{m_{n}}\left(\theta+2 \pi l_{0}-m_{1} \varphi_{1}^{0}-\ldots m_{n-1} \varphi_{n-1}^{0}\right) \in\left(-\frac{\pi}{2}, \frac{\pi}{2}\right), \\
& \varphi_{j}^{0} \in\left(-\frac{\pi}{2}, \frac{\pi}{2}\right) \text { for all } j=\overline{1, n-1} .
\end{aligned}
$$

Hence,

$$
\frac{1}{m_{n}}\left(\theta+2 \pi l_{0}-m_{1} \varphi_{1}^{0}-\ldots m_{n-1} \varphi_{n-1}^{0}\right)-\varphi_{j}^{0}=0, \quad j=\overline{1, n-1} .
$$

We have obtained a system of linear algebraic equations for $\varphi_{1}^{0}, \ldots, \varphi_{n-1}^{0}$. This system has the unique solution

Now we find $\varphi_{n}^{0}$ :

$$
\varphi_{1}^{0}=\ldots=\varphi_{n-1}^{0}=\frac{\theta+2 \pi l_{0}}{|m|}
$$

$$
\varphi_{n}^{0}=\frac{1}{m_{n}}\left(\theta+2 \pi l_{0}-\left(|m|-m_{n}\right) \frac{\theta+2 \pi l_{0}}{|m|}\right)=\frac{\theta+2 \pi l_{0}}{|m|} .
$$

Hence, identities 4.5 hold true. The proof is complete.

\section{REPRESENTATION OF BOUNDED SOLUTION}

Here prove Theorem 2.3 on representing a bounded solution to equation (2.6) by formula (2.10). Let $f \in C_{0}$, the numbers $\alpha_{1}, \ldots, \alpha_{n}$ be positive and condition (2.8) is satisfied. Let us check that if the function $G$ defined by formula (2.11) is absolutely integrable in the domain $x_{1}>0, \ldots, x_{n}>0$, then the function $u$ defined by formula $(2.10)$ is a bounded solution to equation (2.6).

It is obvious that the absolute integrability of $G$ implies that $u \in C_{0}$. If, in addition, $u$ is a generalized solution of equation (2.6), then by Lemma 3.2 we obtain that $u \in C_{m}$ and $u$ is a bounded solution of equation (2.6). In the case, when $f$ has a compact support, we can straightforwardly check that $u$ is indeed a generalized solution. In the case, when $f$ is an arbitrary function in $C_{0}$, the same is checked as follows:

1) As in the proof of Theorem 2.1, we construct a sequence of functions $f_{q} \in C_{0}, q=1,2, \ldots$ with compact supports converging uniformly to $f$ in each bounded set $D \in \mathbb{R}^{n}$;

2) Letting $f=f_{q}$ in formula (2.10), we get a generalized solution $u_{q} \in C_{0}$ of equation (2.6);

3 ) It follows from representation 2.10 that the sequence $u_{q}, q=1,2, \ldots$ converges to $u$ uniformly in each bounded set $D \in \mathbb{R}^{n}$;

4) Letting $f=f_{q}, u=u_{q}$ in equation (2.6) and passing to the limit as $q \rightarrow \infty$, we obtain that $u$ is a generalized solution of equation (2.6). Thus, the proof of Theorem 2.3 is reduced to checking the absolute integrability of the function $G$ in the domain $x_{1}>0, \ldots, x_{n}>0$.

We write the function $G$ as

$$
G\left(x_{1}, \ldots, x_{n}\right)=e^{-a_{1} x_{1}-\ldots-a_{n} x_{n}} x_{1}^{m_{1}-1} \ldots x_{n}^{m_{n}-1} z\left(x_{1}^{m_{1}} \ldots x_{n}^{m_{n}}\right),
$$

where

$$
z(t)=\sum_{k=0}^{\infty} \frac{(b \cdot t)^{k}}{\left(m_{1}(k+1)-1\right) ! \ldots\left(m_{n}(k+1)-1\right) !} .
$$

It is obvious that the function $z(t)$ is well-defined and infinitely differentiable on the interval $(-\infty,+\infty)$. Let us estimate the growth rate of $|z(t)|$ for large positive $t$. The following lemma is true. 
Lemma 5.1. There exist positive numbers $M$ and $\beta$ depending only on $n$ and $m_{1}, \ldots, m_{n}$ such that for all $t \geqslant 1$ and $l=0,1, \ldots,|m|-1$ the estimate

$$
\left|z^{(l)}(t)\right| \leqslant M t^{(\beta-l(|m|-1)) /|m|} e^{\lambda_{m} \sqrt[|m|]{t}}
$$

holds true, where

$$
\lambda_{m}=\frac{|m| R_{|m|}(b)}{\sqrt[|m|]{m_{1}^{m_{1}} \cdots m_{n}^{m_{n}}}} .
$$

We employ Lemma 5.1 to estimate $\left|G\left(x_{1}, \ldots, x_{n}\right)\right|$ from above for $x_{1}>0, \ldots, x_{n}>0$. It follows from Lemma 5.1 that

$$
e^{-\lambda_{m}} \sqrt[|m|]{t}|z(t)| \leqslant M_{3}\left(1+t^{\beta /|m|}\right) \quad \text { for all } \quad t \geqslant 0
$$

where $M_{3}>0$ and $M_{3}$ is independent of $t$.

Taking into consideration condition $(2.8)$, we choose $\varepsilon>0$ so that the inequality

$$
R_{|m|}(b)<\left(\left(\alpha_{1}-\varepsilon\right)^{m_{1}} \ldots\left(\alpha_{n}-\varepsilon\right)^{m_{n}}\right)^{1 /|m|}
$$

holds true. Then for all $x_{1}>0, \ldots, x_{n}>0$ we have

$$
\begin{aligned}
\left(\alpha_{1}-\varepsilon\right) & x_{1}+\ldots+\left(\alpha_{n}-\varepsilon\right) x_{n}=m_{1}\left(\frac{\alpha_{1}-\varepsilon}{m_{1}} x_{1}\right)+\ldots+m_{n}\left(\frac{\alpha_{n}-\varepsilon}{m_{n}} x_{n}\right) \\
& \geqslant|m|\left(\left(\frac{\alpha_{1}-\varepsilon}{m_{1}} x_{1}\right)^{m_{1}} \cdot \ldots \cdot\left(\frac{\alpha_{n}-\varepsilon}{m_{n}} x_{n}\right)^{m_{n}}\right)^{1 /|m|} \\
& >\frac{|m| R_{|m|}(b)}{\sqrt[|m|]{m_{1}^{m_{1}} \ldots m_{n}^{m_{n}}}}\left(x_{1}^{m_{1}} \cdot \ldots \cdot x_{n}^{m_{n}}\right)^{1 /|m|}=\lambda_{m}\left(x_{1}^{m_{1}} \cdot \ldots \cdot x_{n}^{m_{n}}\right)^{1 /|m|} .
\end{aligned}
$$

We estimate $\left|G\left(x_{1}, \ldots, x_{n}\right)\right|$ by employing inequality $(5.5)$ :

$$
\begin{aligned}
\left|G\left(x_{1}, \ldots, x_{n}\right)\right| & =e^{-\varepsilon\left(x_{1}+\ldots+x_{n}\right)} e^{-\left(\alpha_{1}-\varepsilon\right) x_{1}-\ldots-\left(\alpha_{n}-\varepsilon\right) x_{n}} x_{1}^{m_{1}-1} \ldots x_{n}^{m_{n}-1}\left|z\left(x_{1}^{m_{1}} \ldots x_{n}^{m_{n}}\right)\right| \\
& <e^{-\varepsilon x_{1}} x_{1}^{m_{1}-1} \ldots e^{-\varepsilon x_{n}} x_{n}^{m_{n}-1} e^{-\lambda_{m}} \sqrt[|m|]{x_{1}^{m_{1}} \ldots x_{n}^{m_{n}}}\left|z\left(x_{1}^{m_{1}} \ldots x_{n}^{m_{n}}\right)\right| \\
& \leqslant M_{3} e^{-\varepsilon x_{1}} x_{1}^{m_{1}-1} \ldots e^{-\varepsilon x_{n}} x_{n}^{m_{n}-1}\left(1+\left(x_{1}^{m_{1}} \ldots x_{n}^{m_{n}}\right)^{\beta /|m|}\right) .
\end{aligned}
$$

This implies the absolute integrability of the function $G$ in the domain $x_{1}>0, \ldots, x_{n}>0$.

The proof of Lemma 5.1 will follow the next lemma.

Lemma 5.2. The function $z(t)$ defined by formula (5.2) satisfies the identity

$$
\sum_{l=1}^{|m|} p_{l} t^{l-1} z^{(l)}(t) \equiv b z(t)
$$

where $p_{0}=0, p_{1}, \ldots, p_{|m|}$ are the coefficients of the expansion of the polynomial

$$
Q(z)=\prod_{j=1}^{n}\left(m_{j} z+m_{j}\right)\left(m_{j} z+m_{j}+1\right) \ldots\left(m_{j} z+2 m_{j}-1\right)
$$

into the interpolation Newton polynomial [12] by the nodes $-1,0,1, \ldots,|m|-1$ :

$$
Q(z)=\sum_{l=1}^{|m|} p_{l}(z+1) z \ldots(z-l+2) .
$$


Proof. Expansion (5.7) implies the following identities:

$$
\begin{aligned}
& \sum_{l=1}^{k+1} p_{l}(k+1) k \ldots(k-l+2)=Q(k), \quad k=0,1, \ldots,|m|-1, \\
& \sum_{l=1}^{|m|} p_{l}(k+1) k \ldots(k-l+2)=Q(k), \quad k=|m|,|m|+1, \ldots .
\end{aligned}
$$

This implies

$$
\begin{aligned}
& \sum_{l=1}^{|m|} p_{l} t^{l-1} z^{(l)}(t)=b \sum_{l=1}^{|m|} p_{l} \sum_{k=l-1}^{\infty} \frac{(b t)^{k}(k+1) k \ldots(k-l+2)}{\left(m_{1}(k+2)-1\right) ! \ldots\left(m_{n}(k+2)-1\right) !} \\
&=b \sum_{l=1}^{|m|} p_{l}\left(\sum_{k=l-1}^{|m|-1}+\sum_{k=|m|}^{\infty}\right) \frac{(b t)^{k}(k+1) k \ldots(k-l+2)}{\left(m_{1}(k+1)-1\right) ! \ldots\left(m_{n}(k+1)-1\right) ! \cdot Q(k)} \\
&=b \sum_{k=0}^{|m|-1} \frac{(b t)^{k}(k+1) k \ldots(k-l+2)}{\left(m_{1}(k+1)-1\right) ! \ldots\left(m_{n}(k+1)-1\right) ! \cdot Q(k)} \sum_{l=1}^{k+1} p_{l}(k+1) k \ldots(k-l+2) \\
&+b \sum_{k=|m|}^{\infty} \frac{(b t)^{k}(k+1) k \ldots(k-l+2)}{\left(m_{1}(k+1)-1\right) ! \ldots\left(m_{n}(k+1)-1\right) ! \cdot Q(k)} \sum_{l=1}^{|m|} p_{l}(k+1) k \ldots(k-l+2) \\
&= b z(t) .
\end{aligned}
$$

The proof is complete.

Proof of Lemma 5.1. It follows from identity (5.6) that the vector function $y(t)=$ $\left(z(t), z^{\prime}(t), \ldots, z^{(|m|-1)}(t)\right)^{\top}$ is a solution to the system of differential equations

$$
y^{\prime}(t)=B(t) y(t), \quad t>0
$$

where

$$
B(t)=\left(\begin{array}{cccccc}
0 & 1 & 0 & \cdots & 0 & 0 \\
0 & 0 & 1 & \cdots & 0 & 0 \\
\cdot & \cdot & \cdot & \cdots & . & \cdot \\
0 & 0 & 0 & \cdots & 0 & 1 \\
\frac{b}{p_{\left.|m|\right|^{|m|-1}}} & \frac{-p_{1}}{p_{\left.|m|\right|^{|m|-1}}} & \frac{-p_{2}}{\left.p_{|m|}\right|^{|m|-2}} & \cdots & \frac{-p_{|m|-2}}{p_{|m|^{2}}} & \frac{-p_{|m|-1}}{p_{|m|}}
\end{array}\right), \quad p_{|m|}=m_{1}^{m_{1}} \ldots m_{n}^{m_{n}} .
$$

In system (5.8) we make the change

$$
y(t)=\left.C(\tau) u(\tau)\right|_{\tau=t^{1 /|m|}},
$$

where

$$
C(\tau)=\operatorname{diag}\left(1, c_{1}(\tau), \ldots, c_{|m|-1}(\tau)\right), \quad c_{l}(\tau)=\left(|m| \tau^{|m|-1}\right)^{-l}, \quad l=1, \ldots,|m|-1 .
$$

As a result, we obtain the system of differential equations

$$
u^{\prime}(\tau)=D(\tau) u(\tau), \quad \tau>0,
$$

where

$$
D(\tau)=|m| \tau^{|m|-1} C^{-1}(\tau) B\left(\tau^{|m|}\right) C(\tau)-C^{-1}(\tau) C^{\prime}(\tau) .
$$

We calculate $D(\tau)$ :

$$
D(\tau)=D_{0}+D_{1}(\tau),
$$


where the matrix $D_{1}(\tau)$ satisfies the condition $\left|D_{1}(\tau)\right| \leqslant M_{4} \tau^{-1}$ as $\tau \geqslant 1$, and the matrix $D_{0}$ is determined by the formula

$$
D_{0}=\left(\begin{array}{cccccc}
0 & 1 & 0 & \ldots & 0 & 0 \\
0 & 0 & 1 & \ldots & 0 & 0 \\
. & . & . & \ldots & . & . \\
0 & 0 & 0 & \ldots & 0 & 1 \\
\frac{b|m|^{|m|}}{p_{|m|}} & 0 & 0 & \ldots & 0 & 0
\end{array}\right) .
$$

The eigenvalues of the matrix $D_{0}$ are $|m|$-th roots of the number $b|m|^{|m|} / p_{|m|}$ :

$$
w_{k}=|m|\left(\frac{b}{p_{|m|}}\right)^{1 /|m|} e^{i(\theta+2 \pi(k-1)) /|m|}, \quad k=1, \ldots,|m|,
$$

where $\theta$ is the argument of the complex number $b$. To each eigenvalue $w_{k}$, the eigenvector $\left(1, w_{k}, \ldots, w_{k}^{|m|-1}\right)^{\top}$ is associated. By these eigenvectors we form the matrix

$$
W=\left(\begin{array}{ccc}
1 & \ldots & 1 \\
w_{1} & \ldots & w_{|m|} \\
\dot{m} & \ldots & \cdot \\
w_{1}^{|m|-1} & \ldots & w_{|m|}^{|m|-1}
\end{array}\right) .
$$

It is easy to check the identity $W^{-1} D_{0} W=\Lambda$, where $\Lambda=\operatorname{diag}\left(w_{1}, \ldots, w_{|m|}\right)$. Making the change

$$
u(\tau)=W v(\tau)
$$

in system (5.11), we obtain the system

$$
v^{\prime}(\tau)=\left(\Lambda+W^{-1} D_{1}(\tau) W\right) v(\tau), \quad \tau>0 .
$$

For the coordinates $v_{j}(\tau), j=1, \ldots,|m|$, of the vector function $v(\tau)$ we have

$$
v_{j}^{\prime}(\tau)-w_{j} v_{j}(\tau)=e_{j 1}(\tau) v_{1}(\tau)+\ldots+e_{j|m|}(\tau) v_{|m|}(\tau),
$$

where $\left|e_{j l}(\tau)\right| \leqslant \beta_{0} \tau^{-1}$ for all $\tau \geqslant 1, j, l=1, \ldots,|m|$. We multiply each differential equation by $\exp \left(-w_{j} \tau\right)$ and then we integrate from 1 to $\tau$ :

$$
v_{j}(\tau) e^{-w_{j} \tau}=v_{j}(1) e^{-w_{j}}+\int_{1}^{\tau}\left(e_{j 1}(\xi) v_{1}(\xi)+\ldots+e_{j|m|}(\xi) v_{|m|}(\xi)\right) e^{-w_{j} \xi} d \xi
$$

We estimate $\left|v_{j}(\tau)\right|$ as $\tau \geqslant 1$ :

$$
\left|v_{j}(\tau)\right| \leqslant M_{5} e^{\lambda_{m} \tau}+\beta_{0} \int_{1}^{\tau}\left(\left|v_{1}(\xi)\right|+\ldots+\left|v_{|m|}(\xi)\right|\right) e^{\lambda_{m}(\tau-\xi)} \xi^{-1} d \xi
$$

where $\lambda_{m}=\max \left(\operatorname{Re}\left(w_{1}\right), \ldots, \operatorname{Re}\left(w_{|m|}\right)\right)$. This implies:

$$
e^{-\lambda_{m} \tau} \sum_{j=1}^{|m|}\left|v_{j}(\tau)\right| \leqslant M_{5}|m|+\beta_{0}|m| \int_{1}^{\tau} \xi^{-1} e^{-\lambda_{m} \xi} \sum_{j=1}^{|m|}\left|v_{j}(\xi)\right| d \xi \quad \text { as } \quad \tau \geqslant 1 .
$$

By Grönwall lemma [1] this inequality yields the estimate

$$
e^{-\lambda_{m} \tau} \sum_{j=1}^{|m|}\left|v_{j}(\tau)\right| \leqslant M_{5}|m| \tau^{\beta_{0}|m|} \quad \text { as } \quad \tau \geqslant 1 .
$$

In view of the obtained estimate, changes (5.12), (5.9) and formula (5.10), it is easy to get estimates (5.3). The proof is complete. 


\section{BIBLIOGRAPHY}

1. B.P. Demidovich. Lectures on mathematical stability theory. Nauka, Moscow (1967). (in Russian).

2. E. Muhamadiev. To theory of differential equations in the space of bounded generalized functions // Izv. AN Tadzh. SSR. 110:4, 77-80 (1988). (in Russian).

3. G.E. Shilov. Mathematical analysis. V. 2: Elementary functional analysis. Nauka, Moscow (1965). [M.I.T. Press, Cambridge, Mass. (1974).]

4. E. Muhamadiev, S. Baizaev. Bounded solutions of hyperbolic equations with constant coefficients // Izv. AN RT. 142:1, 20-25 (2011). (in Russian).

5. E. Mukhamadiev, A.N. Naimov, A.Kh. Sattorov. Bounded solutions of a class of hyperbolic equations on the plane // Differ. Uravn. 52:1, 86-93 (2016). [Differ. Equ. 52:1, 84-91 (2016).]

6. V.P. Palamodov. Linear differential operators with constant coefficients. Nauka, Moscow (1967). [Springer, New York (1970).]

7. V.Z. Meshkov. Weight differential inequalities and their applications to estimates of the decrease order at infinity of the solutions to elliptic equations of the second order // Trudy Matem. Inst. Steklov. 190, 139-158 (1989). [Proc. Steklov Inst. Math. 190, 145-166 (1992).]

8. V.S. Vladimirov. Equations of mathematical physics. Nauka, Moscow (1981). [Pure Appl. Math. 3. Marcel Dekker, New York (1971).]

9. L. Bers, F. John, M. Schechter. Partial differential equations. Interscience Publisher, New York (1964).

10. A.N. Kolmogorov, S.V. Fomin. Introductory real analysis. Fizmatlit, Moscow (2004). [PrenticeHall, Inc. Englewood Cliffs (1970).]

11. A.G. Kurosh. A kurs of higher algebra. Nauka, Moscow (1968). (in Russian).

12. B.P. Demidovic, I.A. Maron. Computational mathematics. Nauka, Moscow (1966). [Mir Publishers, Moscow (1976).]

Ergashboj Mirzoevich Muhamadiev,

Vologda State University,

Lenin str., 15,

160000, Vologda, Russia

E-mail: emuhamadiev@rambler.ru

Alizhon Nabidzhanovich Naimov,

Vologda State University,

Lenin str., 15,

160000, Vologda, Russia

Vologda Institute of Law and Economics,

Schetinina str., 2,

160002, Vologda, Russia

E-mail: nan670rambler.ru

Akhmad Khasanovich Sattorov,

Khujand State University

named after Academician B. Gafurov,

Mavlonbekov passage, 1 ,

735700, Khudjand, Republic of Tajikistan

E-mail: shuhrat27@mail.ru 